Historic, Archive Document

Do not assume content reflects current scientific knowledge, policies, or practices. 



\section{Spring of 1928}

Semi-Centennial Price List

of

GENERAL NURSERY STOCK

DIRECT FROM THE WESTERN NEW YORK NURSERIES

of

King Brothers Nurseries INCORPORATED

DANSVILLE - NEW YORK

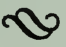

\section{To Our Select Trade and Mail Order Patrons:}

WVE submit herewith our Semi-Centennial Price List offerW ing a complete assortment of nursery stock for orchard, garden or lawn. Economies in large-scale production, the elimination of expensive advertising and printing enable us to give the benefit of wholesale direct from grower to planter prices to our thousands of regular patrons throughout the country. Here is the opportunity you have been looking for to fill out your orchard or fruit garden and beautify your home grounds with shrubs and ornamental stock at wholesale prices-the actual cost of production plus one small profit. No gaudy display will be found in this price list, but offers of real value at prices that will mean a big saving to you on every tree or shrub you buy. Remember, we have been established in the Genesee Valley since 1878. We have reached the half-century mark. King's trees have been planted in every state in the Union. The quality of our stock and the character of our service is attested by thousands of pleased patrons throughout the United States. You can buy no better trees elsewhere at any price than the stock we offer you in this Semi-Centennial Price List. Order early. Orders filled in the rotation in which they are received at planting time.

We take this opportunity to thank our thousands of patrons and friends for their liberal patronage in the past and the recommendation of our stock to their friends. We look forward to the greatest volume of business in our history this year through the recommendations of our friends and our great reduction in prices.

King Brothers Nurseries, Inc. 


\section{TERMS AND CONDITIONS OF SALE}

Shipping Season: Usually opens about March 25 th and continues to the middle of June.

Rates: Less than 10 trees or shrubs at each rate; 10 to 50 at the 10 rate; 50 or more at the 100 rate, provided there is not less than 10 of any one variety.

Terms: Ten per cent discount on all cash orders mailed before March 15th. Six per cent discount after that date and until April 15th. Three per cent discount after April 15th. We will ship trees and plants C.O.D. providing 25 per cent of the total of your order is sent cash with the order. The discount does not apply on C.O.D. orders.

How to Remit: You may send us the money by Registered Letter, Post Office Money Order, Draft or your personal check.

Freight and Express Charges paid by you on all stock bought at this special sale price.

OUR GUARANTEE: First-We guarantee our trees to reach you in a live, thrifty condition, and to be free from all plant diseases.

Second-It is obviously to our advantage to have all stock that leaves our nursery true to name and satisfactory to the purchaser. In the event that any nursery stock sold by us should prove untrue to name under which it is sold, we hereby agree, on proof of such untrueness to name, to replace the original order or to refund the purchase price. Except as to such liability and in respect to all nursery stock sold by us, we give no warranty, expressed or implied, as to description, quality, productiveness, or any other matter.

No. 2093

\section{STATE OF NEW YORK \\ DEPARTMENT OF AGRICULTURE AND MARKETS BERNE A. PYRKE, Commissioner}

Certificate of Inspection of Nursery Stock

THIS IS TO CERTIFY that the nursery stock of KING BROTHERS NURSERIES, Inc., of Dansville, County of Livingston, State of New York, has been duly examined in compliance with the Agriculture and Markets Law and has been found to be apparently free from all injurious insects and plant diseases. This certificate is valid until September 1, 1928.

Dated, Albany, N. Y., September 19, 1927.

BERNE A. PYRKE, Commissioner.

\section{BEARING AGE TREES}

\section{Seven to Eight Feet Tall for Quick Results}

Reduce the high cost of living by making every available foot of space in your garden produce something. We have prepared our bearing age grade of trees especially for the suburban gardener and family with the small back-yard where quick results after planting both as to shade and fruiting, as well as the good appearance of the back yard are desired.

Note this astonishingly low price on all the varieties listed below of Bearing Age Trees.

Bearing Age grade, 7 to 8 feet tall, 98c each, $\$ 8.00$ per $10, \$ 70.00$ per 100 . Varieties of Apples: Yellow Transparent, Duchess of Oldenburg, Wealthy, Winter 
BEARING AGE TREES-Continued

Banana, King, Rome Beauty, Delicious, Northern Spy, Ben Davis, Jonathan, Rhode Island Greening, Stayman's Winesap, N. W. Greening, Gravenstein, Stark, Wagener, Baldwin.

Varieties of Pears: Bartlett, Seckel, Anjou, Kieffer, Duchess d'Angouleme, Sheldon, Clapp's Favorite, Flemish Beauty, Lawrence.

Varieties of Cherries: Bing, Black Tartarian, Gov. Wood, Napoleon, May Duke, Montmorency, Early Richmond, English Morello.

Varieties of Plums: Abundance, Burbank, October Purple, Red June, Satsuma, Wickson, Bradshaw, French Damson, German Prune, Lombard, Shropshire Damson.

Varieties of Peaches: Elberta, Early Crawford, Late Crawford.

\section{SPECIAL GARDEN COLLECTION GREAT PRICE REDUCTION OFFER}

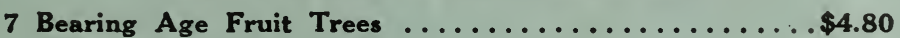
I Baldwin Apple, I Bartlett Pear, I Burbank Plum, I Lombard Plum, I Black Tartarian Cherry, 1 Montmorency Cherry, I Crawford Peach.

All 7 to 8 feet for only $\$ 4.80$. Express Collect.

SPECIAL GARDEN GRAPE VINE ARBOR

6 Beautiful Large Grape Vines, \$1.00-Parcel Post Prepaid.

\section{EIGHT POPULAR SHRUBS THAT BLOOM ALL SUMMER}

Forsythia Fortunei

Blooms in April

Honeysuckle-Red Tartarian

Blooms May to July

Deutzia-Pride of Rochester

Blooms in May

Weigelia Rosea

Blooms in June

Price For Above Collection, 8 Shrubs, $\$ 3.60$ - Express Collect
Spirea Van Houttei

Blooms in May-June

Spirea Anthony Waterer

Blooms in July-August

Syringa, Mock Orange

Blooms in May-June

Hydrangea P. G.

Blooms in August-October

“50 Years' Experience and Record for Service Back of Our Trees"

\section{PRICE LIST}

King's Book, containing seventy-two pages-all about fruits and flowers and their care-sent you for $10 \mathrm{c}$ in stamps. A $20 \mathrm{c}$ rebate slip which we will send you with the book will entitle you to a grape vine free with your order.

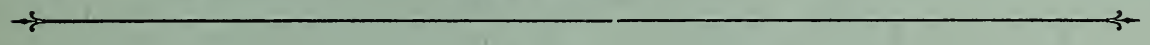

\section{APPLES}

Prices on all the varieties

\section{Height}

Largest size, 2-year. .....6 to $7 \mathrm{ft}$.

Medium size, 2-year ....... to $6 \mathrm{ft}$.

Smaller size, 2-year ......4 to $5 \mathrm{ft}$.

$\underset{\mathbf{X X X}}{\mathbf{X X}}$

Each

Per 10

6.00

$\begin{array}{ll}.50 & 4.40 \\ .40 & 3.00\end{array}$

48.00

36.00

26.00

Largest size, 1-year.........3ft. and up. Sturdy buds. $70 \quad 6.00 \quad 48.00$

SUMMER VARIETIES: Bough, Early Harvest, Red Astrachan, Yellow Transparent, Williams Early Red.

AUTUMN VARIETIES: Alexander, Duchess of Oldenburg, Fall Pippin, Gravenstein, Maiden's Blush, Rambo, Pound Sweet.

WINTER VARIETIES: Baldwin, Banana, Ban Davis, Cortland, Delicious, Fameuse, Gano, Grimes Golden, Hubbardston, Jonathan, King, Mclntosh Red, Northern Spy, North Western Greening, Opalescent, Rhode Island Greening, Rome Beauty, 


\title{
APPLES-Continued
}

Roxbury Russet, Smokehouse, Stark, Stayman's Winesap, Spitzenburg, Sutton Beauty, Tolman Sweet, Twenty Ounce, Wagener, Wealthy, Winesap, Wolf River, Yellow Newtown, York Imperial.

CRAB APPLES: Gen. Grant, Hyslop, Transcendent.

SWEET CHERRIES

Prices on all the varieties

\section{Height}

Each Per $10 \quad$ Per 100

Largest size, 2-year ......6 to 7 ft. XXX $\mathbf{X X} \quad \$ 6.75 \quad \$ 58.00$

Medium size, 2-year .......5 to 6 ft. $X X$

Smaller size, 2-year ......4 to 5 ft. $X \quad \begin{array}{llll} & \mathbf{f 5} & 4.75 & 39.00\end{array}$

VARIETIES: Black Tartarian, Bing, Governor Wood, Lambert, Napoleon, Schmidt's Bigarreau, Windsor, Yellow Spanish.

\section{SOUR CHERRIES}

\section{Height}

Largest size, 2-year ......6 to $7 \mathrm{ft}$.

Medium size, 2-year .......5 to $6 \mathrm{ft}$.

Smaller size, 2-year .......4 to $5 \cdot \mathrm{ft}$.

$\underset{\mathbf{X X}}{\mathbf{X X X}}$

\author{
Each
}

Per 10

Per 100

$\$ .75$

$\$ 6.40$

$\$ 50.00$

$.60 \quad 5.60$

40.00

$.45 \quad 4.00 \quad 38.00$

Montmorency.

\section{PEACHES}

Height

Largest size, 1-year ......5 to $6 \mathrm{ft}$.

Medium size, 1-year ......4 to $5 \mathrm{ft}$.

Smaller size, 1-year .......3 to $4 \mathrm{ft}$.

$\begin{array}{lrrr} & \text { Each } & \text { Per 10 } & \text { Per 100 } \\ \text { XXX } & \$ .45 & \$ 3.60 & \$ 29.00 \\ \text { XX } & .40 & 3.00 & 24.00 \\ \text { X } & .30 & 2.00 & 18.00\end{array}$

Large

VARIETIES: Belle of Georgia, Captain Ede, Carman, Chair's Choice, Cham. pion, Crosby, Crawford Early, Crawford Late, Elberta, Fitzgerald, Greensboro, Hale, Hill's Chili, Hiley, Kalamazoo, Mountain Rose, Niagara, Old Mixon, Rochester, Sa]. way, Beer's Smock, Triumph, Wonderful, Yellow St. John.

\section{STANDARD PEARS}

$$
\text { Height Each Per } 10 \text { Per } 100
$$

Largest size, 2-year .......6 to $7 \mathrm{ft} . \quad$ XXX $\$ .70 \quad \$ 6.00 \quad \$ 55: 00$

Medium size, 2-year ......5 to $6 \mathbf{f t}$. XX $\quad .60 \quad 5.50 \quad 48.00$

Smaller size, 2-year .......4 to $5 \mathrm{ft}$. $\mathrm{X} \quad \mathbf{4 0} \quad \mathbf{3 . 5 0} \quad \mathbf{3 2 . 0 0}$

VARIETIES: Anjou, Bartlett, Beurre Bosc, Beurre Clairgeau, Clapp's Favor. ite, Duchess D'Angouleme, Flemish Beauty, Kieffer, Lawrence, Lincoln, Seckel, Sheldon, Vermont Beauty, Wilder, Winter Nelis.

\section{PLUMS}

Height

Largest size, 2-year ......6 to $7 \mathrm{ft}$.

Medium size, 2-year ....... 5 to $6 \mathrm{ft}$.

Smaller size, 2-year .......4 to $5 \mathrm{ft}$.

VARIETIES: Abundance,

$\begin{array}{lrrr} & \text { Each } & \text { Per 10 } & \text { Per } 100 \\ \text { XXX } & \$ .60 & \$ 5.00 & \$ 44.00 \\ \text { XX } & .50 & 4.00 & 32.00 \\ \text { X } & .40 & 3.50 & 24.00\end{array}$
Wickson, Arctic, Bradshaw, German Prune, Green Gage, Grand Duke, Imperia! Gage, Lombard, Monarch, Pond Seedling, Shippers Pride, Shropshire Damson, Yellow Egg, York State Prune.

\section{QUINCES}

Height Each Per 10 Per 100

Largest size, 2-year ......4 to $6 \mathrm{ft}$. XXX $\$ .70 \quad \$ 6.40 \quad \$ 59.00$

Medium size ...........3 to $4 \mathrm{ft}$. XX $\quad .60 \quad 5.20 \quad \$ 8.00$

Champion, Orange, Meech, Rea's Mammoth.

\section{DWARF APPLE}

\section{Height}

2 to 3-year $\ldots \ldots \ldots \ldots \ldots$ to $6 \mathrm{ft}$

2 to 3 -year $\ldots \ldots \ldots \ldots \ldots \ldots \ldots \ldots$ to $4 \mathrm{ft}$.

$\begin{array}{rrr}\text { Each } & \text { Per } 10 & \text { Per } 100 \\ \$ .70 & \$ 5.50 & \$ 45.00 \\ .65 & 4.75 & 35.00\end{array}$

35.00

Duchess of Oldenburg, Fameuse, Mclntosh Red, Wealthy, Winter Banana, Yellow Transparent.

\section{DWARF PEARS}

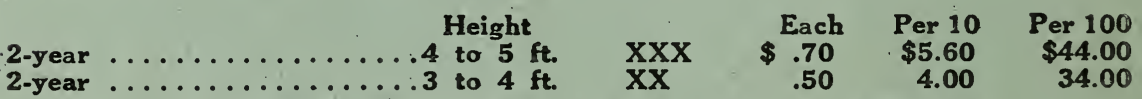

We offer the same dwarf varieties as listed under Standard Pears. 
APRICOTS: Price, 4 to $6 \mathrm{ft.}, 70 \mathrm{c}$ each; $\$ 5.00$ per $10 ; \$ 45.00$ per 100 .

VARIETIES: Alexander, Moorpark, Harris, Orange.

GRAPES-Price on all varieties except Concord.

2 yr. No. I Vines, 20 c each, $\$ 1.90$ per 10, $\$ 18.00$ per 100

Concord, 2 yr. No. 1, $18 \mathrm{c}$ each, $\$ 1.50$ per. 10 , $\$ 10.00$ per 100.

Varieties: Agawam, Brighton, Campbells Early; Catawba, Delaware, Moore's Early, Niagara, Pocklington, Salem, Worden.

CURRANTS: Strong, healthy 2yr. vines, $16 \mathrm{c}$ éach, $\$ 1.40$ per $10, \$ 12.00$ per 100 . Varieties: Cherry, Fay's Prolific, Perfection, Red Dutch, Red Cross, White Grape, Wilder.

BLACK RASPBERRIES: Price, 90c per $25, \$ 3.20$ per $100, \$ 26.50$ per 1000.

Varieties: Cumberland, Gregg, Kansas, Plum Farmer.

RED RASPBERRIES: Price 90 c per 25, \$3.20 per 100, $\$ 26.50$ per 1000 .

Varieties: Cuthbert, Herbert, King, June, Loudon, St. Regis Everbearing.

PURPLE RASPBERRIES: Price, $\$ 1.00$ per $25, \$ 3.50$ per $100, \$ 28.00$ per 1000 .

Varieties: Columbian, Shaffer's Colossal.

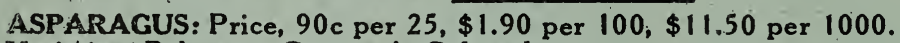

Varieties:' Palmetto, Conover's Colossal.

RHUBARB: Price, $16 \mathrm{c}$ each, $\$ 1.50$ per $10, \$ 10.00$ per 100.

Varieties: Early Scarlet, Myatt's Linnaeus.

BLACKBERRIES: Price, $\$ 1.45$ per $25, \$ 4.40$ per $100, \$ 34.00$ per 1000 .

Varieties: Blowers, Eldorado, Erie, Mersereau, Rathbun, Snyder, Lucretia Dewberry.

COOSEBERRIES: Price, 25c each, \$2.20 per 10, $\$ 20.00$ per 100.

Varieties: Chautauqua, Downing, Houghton, Josselyn.

\section{HARDY FLOWERING SHRUBS}

\section{All Shrubs 48c Each Except as Otherwise Noted}

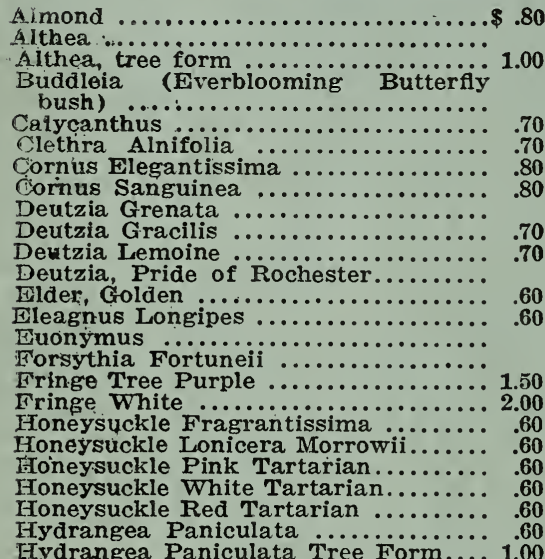

Kerriá $\ldots \ldots \ldots \ldots \ldots \ldots \ldots \ldots \ldots \ldots \ldots . . .80$

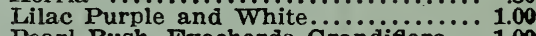

Pearl Bush, Exochorda Grandifiora... 1.00

Plum Triloba ................. 1.50

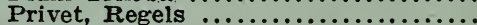

Quince Japonica ...................60

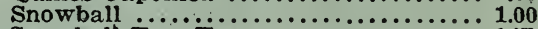

Snowball Tree Form ................. 1.50

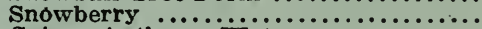

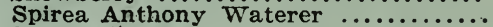

Spirea Arguta ..................60

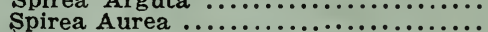

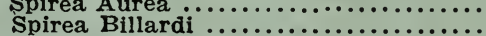

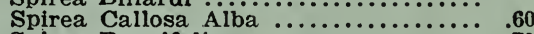

Spirea Prunifolia .................

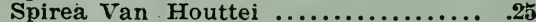

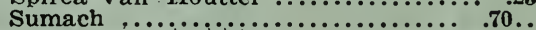

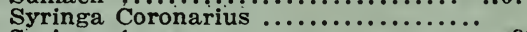

Syringa Aureus ..................... 80

Syringa Virginal $\ldots \ldots \ldots \ldots \ldots \ldots \ldots \ldots \ldots \ldots$

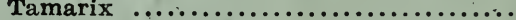

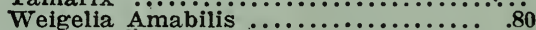

Weigelia Candida $\ldots \ldots \ldots \ldots \ldots \ldots \ldots \ldots \ldots . .8 \ldots \ldots \ldots$

Weigelia Floribunda $\ldots \ldots \ldots \ldots \ldots \ldots \ldots \ldots \ldots . \quad 8 \ldots$

Weigelia Eva Rathke ............... 80

Weigelia Rosea ................. .80

\section{HARDY CLIMBING VINES}

Grergreen Bitter Sweet ..........\$ .50

Cinnamon vine $\ldots \ldots \ldots \ldots \ldots \ldots \ldots \ldots \ldots . .50$

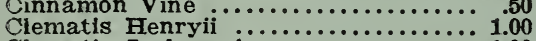

Clematis Jackmani ................. 1.00

Clematis Paniculata $\ldots \ldots \ldots \ldots \ldots \ldots \ldots \ldots . . \ldots \ldots$

Dutchman's Pipe ................. 1.40

Honeysuckle, Hall's Japan ........... .50

Iyy, American $\ldots \ldots \ldots \ldots \ldots \ldots \ldots \ldots \ldots . .50$

Ivy, Bosten . . . . . . . . . . . . . . . . .40

Ivy, English $\ldots \ldots \ldots \ldots \ldots \ldots \ldots \ldots \ldots \ldots \ldots \ldots \ldots \ldots \ldots \ldots . .70$

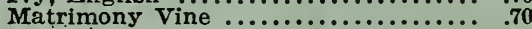

Trumpet Vine .................. 50

Wistaria Purple $. . \ldots \ldots \ldots \ldots \ldots \ldots \ldots \ldots . .90$

Wistaria White $\ldots \ldots \ldots \ldots \ldots \ldots \ldots \ldots \ldots \ldots . .90 \ldots \ldots$

\section{ORNAMENTAL AND SHADE TREES}

Ailanthus, $6-8 \mathrm{ft} . \ldots \ldots \ldots \ldots \ldots \ldots \ldots 1.60$ Ash, American White, $6-8 \mathrm{ft} . \ldots \ldots \ldots 1.20$ Birch (Cut-Leaved Weeping) $3-5 \mathrm{ft}$. 2.70 Birch (European White), 6-8 ft. ......2.10 Catalpa Bungei, 5-6 ft. .............. 1.90 Catalpa Speciosa, 6-8 ft. ............. 1.00 Cherry, Japan Weeping, $4-5$ ft. ..........30 Crab, Bechtel's Flowering, 2-3 ft...... 1.20 Cornus (White Flowering), 2-3 ft. ...6.50 Fim (American), 6-8 ft. ............. 1.40 Judas Tree, $3-4$ ft. $\ldots \ldots \ldots \ldots \ldots \ldots \ldots \ldots . .1 .40$ Larch, European, $3-4$ ft. ............... 1.50
Laburnum (Golden Chain), 4-5 ft. .... 1.80 Linden (American), 6-8 $\mathrm{ft} . \ldots \ldots \ldots \ldots .2 .30$ Linden (European), 6-8 ft. ........ 2.50 Maple, Ash Leaf, 6-8 ft. ............. 1.00 Maple, Norway, 6-8 ft. ............... 3.00 Maple, Silver Leaf, 6-8 $\mathrm{ft} . \ldots \ldots \ldots \ldots \ldots \ldots .1 .00$ Maple, Scarlet, 6-8 ft. ................ Maple, Sugar or Rock, $6-8 \mathrm{ft}$.......... 3.00 Maple, Wier's Cut-eaved, 6-8 ft. ..... 1.80 Maple, Japan (Blood Leaf), 12 to 15 in. .................................... Mountain Ash (Ämerican), $6-8$ $\mathrm{ft} . . . .8 .8 .50$ 
Ornamental and Shade Trees-Continued

Mountain Ash (Weeping) 1 yr hds. 6.00

Mulberry, Downing, 5-6 ft. .......... 1.70

Mulberry, Russian, 6-8 ft. ........... 1.20

Mulberry, Tea's Weeping, $i$ yr. hds... 3.50

Oak; American white, 6-8 ft ........ 3.50

Oak, Scarlet, 4-6 ft. .............. 2.50

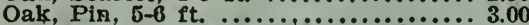

Peach (Double Rose Flowering), $2-3$.

ft. ........................90

Plane, Öriental, $6-8 \mathrm{ft} . . . \ldots \ldots \ldots \ldots . .2 .50$

\section{NUT TREES}

American Filbert, $3-4$ ft. ...........\$1.20

English Filbert, 3-4 ft. .............1.20

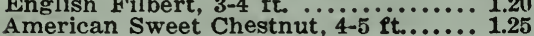

Spanish Chestnut, $4-5 \mathrm{ft}$. $\ldots \ldots \ldots \ldots \ldots .1 .25$

\section{EVERGREENS}

Arbor Vitae, American, 18-24 in. .....\$2.30

Arbor Vitae, Globosa 18-24 in. ....... 3.50

Arbor Vitae, Pyramidal, 18-24 in. .... 5.50

Arbor Vitae, Siberian, 18-24 in.......... 3.20

Arbor Vitae, Tom Thumb, 12-18 in...... 2.50

Arbor Vitae, Golden, 18-24 in........6.50

Juniper, Irish, 18-24 in............ 3.00

Juniper, Savin, 18-24 in............6.00

Juniper, Virginiana, $18-24$ in........... 4.50

Juniper, Retinispora Pisifera, is-24

in. ........................... 3.50

\section{DECORATIVE HEDGES}

Barberry-Thunberg's, 18-24 in...

30 c each; $\$ 2.50$ per $10 ; \$ 20$ per 100

Honey Locust, 18-24 in...........50 each

Osage Orange, 18-24 in...........50 each
Poplar Carolina, 6-8 ft

Poplar, Lombardy, 6-8 ft.

Purple Leaved Plum, $3-4 \mathrm{ft}$.............. 1.00 Salisburia (Maiden Hair), 5-6 ft...... 3.00 Thorn. Paul's Double Scarlet, 8-4 ft... 2.00 Thorn Double White, 3-4 ft. ........ 2.00

Tulip Tree. 6-8 ft. ...............2.20

Willow, Babylonica, $6-8 \mathrm{ft} . \ldots \ldots \ldots \ldots . .150$

Willow, Golden, 4-6 ft. ............. 1.00

willow, Wisconsin Weeping, $6-8 \mathrm{ft} . . .1 .50$

Butternut, 5-6 ft. $\ldots \ldots \ldots \ldots \ldots \ldots \ldots 1.25$

English walnut, $3-4 \mathrm{ft} . \ldots \ldots \ldots \ldots \ldots \ldots .1 .50$

Japan Walnut, $4-5$ ft. $\ldots \ldots \ldots \ldots \ldots \ldots \ldots \ldots . .1 .25$

Black Walnut, 5-6 ft. $\ldots \ldots \ldots \ldots \ldots \ldots \ldots \ldots . .1 .25$

Juniper, Retinispora Pisifera Aurea,

18-24 in. .......................... 30

Spruce, Norway, 18-24 in. .......... 2.00

Spruce, Colorado, 18-24 in. .........9.50

Pine, Austrian, 18-24 in. .........4.50

Pie, Mugho, 18-24 in. ............... 8.50

Pine, Scotch, 18-24 in. ............ 3.00

Rhododendrons, White, Red or Pur-

ple, 18-24 in. .................... 4.25

California Privet, 18-24 in

$\$ 1.00$ per io : $\$ 6.00$ per 100

Amoor River Privet, 18-24 in.

Ibota Privet, 18-24

$\$ 1.50$ per $10 ; \$ 9.00$ per 100

$\$ 1.50$ per $10 ; \$ 9.00$ per 100

\section{ROSES}

First Class, 2 Yr., No. 1 Plants, 62c Each, $\$ 6.00$ Per 10

Old Fashioned June Blooming Varieties:

Alfred Colomb

American Beauty

Anne De Diesbach

Baron de Bonstetten

Clio

Earl of Dufferin

Eugene Furst

Fisher Holmes

Frau Karl Druschki

Gen. Jack

Harrison's Yellow

\section{EVERBLOOMING BUSH ROSES}

Columbia

Crusader

Etoile de France

Francis Scott Key

Gruss An Teplitz

Kaiserin Aug. Victorla

Killarney

La France

\section{(Blooms from June Until Frost)}

Lyon

Mme. Caroline Jesout

My Maryland

Ophelia

Premier

Radiance

Sunburst

\section{CLIMBING ROSES}

Climbing American Beauty

Baltimore Belle

Crimson Rambler

Dorothy Perkins

Dr. W. Van Fleet

Excelsa

Flower of Fairfield

Paul's Scarlet Climber

Silver Moon

Tausendschon

Rosa Rugosa; Blanche Moreau, Moss; Salet, Moss.

Dwarf Polyantha Roses (Pink, White or Crimson Baby Ramblers)

\section{POPULAR OLD-FASHIONED GARDEN FLOWERS}

\section{All Varieties, 25c Each}

Adam's Needle, Achillea, Aconitum, Anchusa, Anemone, Bleeding Heart, Blue Bird Flower (Veronica), Carnations, Columbine, Campanula Cannas, Candytuft, Chrysanthemums, Coreopsis, Daisy Shasta.

(Fox Glove), Eulalia, Funkia, Gaillardia Gladioli, Golden Glow, Gypsophila, Lilies, Myosotis (Forget-me-not), Primulas (Primrose), Hibiscus, Double and Single Hollyhocks, Iris (German and Japan),

Poppy Oriental, Pinks, Phlox, Pyrethrum.

PEONIES-ALI VARIETIES-50c each, as follows: Baron Rothschild, Duchess de Nemours, Duc de Wellington, Festiva Maxima, Humei, Jean d'Arc, Madam de Verneville,
Rubra Superba. 
